\section{Topology for Distributed Inference on Graphs}

Soummya Kar, Saeed Aldosari, and José M. F. Moura

\begin{abstract}
Let $N$ decision-makers collaborate to reach a decision. We consider iterative distributed inference with local intersensor communication, which, under simplifying assumptions, is equivalent to distributed average consensus. We show that, under appropriate conditions, the topology given by the nonbipartite Ramanujan graphs optimizes the convergence rate of this distributed algorithm.
\end{abstract}

Index Terms-Algebraic connectivity, Cayley, consensus algorithm, distributed detection, Laplacian, Ramanujan, random graphs, sensor networks, small-world, spectral graph theory, topology optimization.

\section{INTRODUCTION}

We present the optimal topology design problem for distributed inference in sensor networks; equivalent topology design questions arise in many other applications, including when it is desired to minimize the rate of diffusion of a viral infection, or to maximize (minimize) the spread of a rumor. By topology, we mean the graph that determines the connection or communication channels established among the sensors. We constrain the topology to be sparse, i.e., the number $M$ of channels is much smaller than $N^{2}$, where $N$ is the number of sensors in the network. We further constrain the topology and all graphs in this correspondence to be a connected, simple, undirected graph, [1]. Distributed inference, under simple conditions, is equivalent to average consensus, a widely studied problem, e.g., [2]-[4]. The optimization of weights for fast convergence of consensus on a fixed topology has been studied, e.g., [5], while, simulations showing the impact of specific topologies are presented in [6] and [7]. Our goal is to design the topology that maximizes the speed of convergence of the distributed inference algorithm, which has not received much attention. In general, designing constrained graphs with prescribed properties is a combinatorial problem. The correspondence obtains a somewhat surprising result. Under the appropriate conditions, the constrained design problem has a "closed form" solution-the optimal topology is specified a priori; it is given by a class of expanders, the Ramanujan graphs [8].

Section II describes distributed inference and consensus. Section III presents the Ramanujan graphs as solution to the constrained optimal topology design problem. Section IV shows numerically that the Ramanujan networks are much faster than other topologies. Section V designs new random regular graphs that closely track the performance of Ramanujan topologies but can be constructed for an arbitrary number of sensors, while Ramanujan graphs can only be constructed for a sparse set of integer values of the number of sensors. Finally, Section VI concludes the correspondence. An extended and more detailed version of this correspondence is available on [9].

Manuscript received June 5, 2006; revised February 21, 2008. The associate editor coordinating the review of this manuscript and approving it for publication was Prof. Alle-Jan van der Veen. This work was supported by the DARPA DSO Advanced Computing and Mathematics Program Integrated Sensing and Processing (ISP) Initiative under ARO Grant DAAD19-02-1-0180 and by NSF under Grants ECS-0225449 and CNS-0428404.

S. Kar and J. M. F. Moura are with the Department of Electrical and Computer Engineering, Carnegie Mellon University, Pittsburgh, PA 15213 USA (e-mail: soummyak@ece.cmu.edu; moura@ece.cmu.edu).

S. Aldosari is with Electrical Engineering Department, King Saud University, Riyadh, 11412, Saudi Arabia (e-mail: dosari@ksu.edu.sa).

Color versions of one or more of the figures in this correspondence are available online at http://ieeexplore.ieee.org.

Digital Object Identifier 10.1109/TSP.2008.923536

\section{DISTRIBUTED INFERENCE AND CONSENSUS}

We consider the binary inference between hypotheses $\mathbf{H}_{0}$ and $\mathbf{H}_{1}$, where the data at the sensors, conditioned on the hypothesis $\mathbf{H}_{m}, m=$ 0,1 , are independent, identically distributed (i.i.d.) Gauss random variables $\mathcal{N}\left(\mu_{m}, \sigma^{2}\right), \mu_{0}=-\mu, \mu_{1}=\mu$. The local and global log-likelihood ratios (llr) are

$$
\begin{aligned}
& \mathbf{H}_{m}: \ell_{n} \sim \mathcal{N}\left(\frac{2 \mu \mu_{m}}{\sigma^{2}}, \frac{4 \mu^{2}}{\sigma^{2}}\right), m=0,1, n=1 \cdots N \\
& \mathbf{H}_{m}: \ell=\frac{1}{N} \sum_{n=1}^{N} \ell_{n} \sim \mathcal{N}\left(\frac{2 \mu \mu_{m}}{\sigma^{2}}, \frac{4 \mu^{2}}{N \sigma^{2}}\right), m=0,1 .
\end{aligned}
$$

The global llr can be implemented locally by an iterative distributed consensus algorithm, [2]. It defines the initial state of sensor $n$ as $x_{n}(0)=\ell_{n}$ and, at iteration $i$, each sensor $n$ communicates to its neighbors its current state $x_{n}(i)$, and updates its state by a weighted sum of its neighbors' states. Let $\mathbf{x}(i) \in \Re^{N}$ collect the states of all the $N$ sensors at iteration $i$ and $\mathbf{x}(0)=\left[\ell_{1} \cdots \ell_{N}\right]^{T}$. Assuming noiseless communication

$$
\mathbf{x}(i)=W \mathbf{x}(i-1)=W^{i} \mathbf{x}(0), \quad i \geq 1 .
$$

The entries of row $n, W_{n k}$, of the weight matrix $W$ are nonzero if sensors $k$ and $n$ are neighbors, i.e., there is a direct communication channel among them. Determining the network topology under a constraint on the number of channels is then the problem of designing: i) the structure of $W$, i.e., which ones are its zero entries, assuming a fixed number of nonzero entries, and ii) the actual values of the nonzero entries $W_{n k}$ of $W$. We assume the common condition of a symmetric $W$ with equal weights, [10], i.e., $W=I-\alpha L$. Matrix $L=D-A$ is the Laplacian of the graph, [11], where $A$ is the adjacency matrix ( $A_{n k}=A_{k n}=1$ if there is a communication channel among sensors $n$ and $k$, zero otherwise) and the degree matrix $D$ is diagonal; its entry $d_{n n}$ is the number (degree) of communication channels connecting $n$ to its neighbors. The degree $d_{n n}$ equals the number of 1 's in row $n$ of $A$. The Laplacian $L$ is positive semidefinite, [11]; and, with its eigenvalues ordered as $\lambda_{1}(L)=0 \leq \lambda_{2}(L) \leq \cdots \leq \lambda_{N}(L)$, it follows that $\lambda_{2}(L)>0$ since the topology is connected.

Denote the initial average as $r=\mathbf{1}^{T} \mathbf{x}(0) / N$ where $\mathbf{1}=[1 \cdots 1]^{T}$. Convergence of (3) occurs if

$$
\lim _{i \rightarrow+\infty} W^{i}=\frac{1}{N} \mathbf{1 1}^{T}
$$

We take the optimizing common weight, see [10] for details,

$$
\alpha=\frac{2}{\lambda_{2}(L)+\lambda_{N}(L)} .
$$

Result 1 (Rate of Convergence): For any connected topology, under the equal weights assumption, the state sequence, $\{\mathbf{x}(i)\}_{i \geq 0}$ converges to the average of the initial state, $\mathbf{x}(0)$, with rate $\gamma_{2}<1$, where

$$
\gamma_{2}=\frac{1-\gamma}{1+\gamma}, \quad \text { with } \quad \gamma=\frac{\lambda_{2}(L)}{\lambda_{N}(L)} .
$$

Proof: The proof follows from the fact that the iterative update (3) leads to

$$
\|\mathbf{x}(i)-r \mathbf{1}\| \leq \gamma_{2}^{i}\|\mathbf{x}(0)-r \mathbf{1}\|
$$

so that the convergence rate is $\gamma_{2}$ (see [9] for details).

For distributed inference, let $P_{e}(i, n)$ and $P_{e}$ be the probabilities of error associated with the local and the global minimum probability of error detectors, respectively. Under the conditional independence and Gauss assumptions, the local detectors simply threshold the current 
state $x_{n}(i)$ at sensor $n$ and iteration $i$ and the global detector thresholds the global test statistic $l$.

Result 2 (Distributed Inference): For connected topologies, $\lim _{i \rightarrow+\infty} P_{e}(i, n)=P_{e}, \forall n$.

Proof: It follows from bounding the probabilities of error, noting $\gamma_{2}<1$, and taking the limit $i \rightarrow \infty$

$$
\begin{aligned}
P_{e} & =\operatorname{erfc}\left(\frac{\mu \sqrt{N}}{\sigma}\right) \leq P_{e}(i, n) \\
& =\operatorname{erfc}\left(\frac{\mu \sqrt{N}}{\sigma \sqrt{\left[W^{2 i}\right]_{n n}}}\right) \leq \operatorname{erfc}\left(\frac{\mu \sqrt{N}}{\sigma \sqrt{1+\gamma_{2}^{2 i}(N-1)}}\right) .
\end{aligned}
$$

\section{TOPOLOGY DESIGN: RAMANUJAN GRAPHS}

Topology optimization: To maximize the convergence rate, $\gamma_{2}$ should be made as small as possible, which in turn means that $\gamma$ should be as large as possible, see (6). The topology design is then

$$
\max _{G \in \mathcal{G}} \gamma=\max _{G \in \mathcal{G}} \frac{\lambda_{2}(L)}{\lambda_{N}(L)}
$$

where $\mathcal{G}$ denotes the set of simple connected graphs with $N$ vertices and at most $M$ edges. Equation (9) restates the topology design as optimization of the spectral properties of graphs; see also [9] and [12].

\section{A. Ramanujan Graphs}

We study the spectral properties of $k$-regular graphs, ${ }^{1}$ motivate the consideration of nonbipartite Ramanujan graphs, and, finally, establish their asymptotic (as $N \rightarrow \infty$ ) optimality for the topology design problem in (9) among the class of regular graphs. Then, we argue that the class of nonbipartite Ramanujan graphs are also expected to perform better than the nonregular graphs, thus making them essentially optimal. We first state a well-known result from algebraic graph theory.

Theorem 3 (Alon and Boppana [13], [14]): Let $G=G_{N, k}$ be a $k$-regular graph on $N$ vertices. Denote by $\lambda_{G}(A)$ the absolute value of the largest eigenvalue (in absolute value) of the adjacency matrix $A$ of the graph $G$, which is distinct from $\pm k$; in other words, $\lambda_{G}^{2}(A)$ is the next to largest eigenvalue of $A^{2}$. Then

$$
\liminf _{N \rightarrow \infty} \lambda_{G}(A) \geq 2 \sqrt{k-1}
$$

A second result, [13], also shows that, for an infinite family of $k$-regular graphs $G_{N, k}$, for which the number of nodes $N \rightarrow \infty$, the algebraic connectivity $\lambda_{2}(L)$ is asymptotically bounded by

$$
\liminf _{N \rightarrow \infty} \lambda_{2}(L) \leq k-2 \sqrt{k-1}
$$

Note that (11) is a direct upperbound on the limiting behavior of $\lambda_{2}(L)$ itself, while from (10) we may derive an upperbound on the limiting behavior of $\lambda_{2}(A)$ or of $\lambda_{N}(A)$, depending if $\lambda_{2}(A) \leq\left|\lambda_{N}(A)\right|$ or $\lambda_{2}(A) \geq\left|\lambda_{N}(A)\right|$ in the limit. We consider each of these two cases separately. ${ }^{2}$

1) Consider the family $\mathcal{G}_{1} \subset \mathcal{G}$ of regular graphs for which

$$
\lim _{N \rightarrow \infty} \lambda_{2}(A) \leq \lim _{N \rightarrow \infty}\left|\lambda_{N}(A)\right| .
$$

${ }^{1} \mathrm{~A}$ graph is called $k$-regular if all vertices have the same degree $k$. A bipartite graph is a graph in which the vertex set can be partitioned into two disjoint subsets, such that no two vertices in the same subset are adjacent.

${ }^{2}$ We assume that the limits in (10) and (11) exist. Otherwise, we can extract subsequences, which actually converge.
Since $\lambda_{2}(A)>0$ and $\lambda_{N}(A) \leq 0$, it follows successively

$$
\begin{aligned}
& \lim _{N \rightarrow \infty} \lambda_{N}(A) \leq-2 \sqrt{k-1} \\
& \lim _{N \rightarrow \infty} \lambda_{N}(L) \geq k+2 \sqrt{k-1} .
\end{aligned}
$$

Combining (13) with (11), we get the asymptotic upper bound on $\gamma$

$$
\lim _{N \rightarrow \infty} \gamma(N)=\lim _{N \rightarrow \infty} \frac{\lambda_{2}(L)}{\lambda_{N}(L)} \leq \frac{k-2 \sqrt{k-1}}{k+2 \sqrt{(k-1)}} .
$$

2) Consider the family $\mathcal{G}_{2} \subset \mathcal{G}$ of regular graphs for which

$$
\lim _{N \rightarrow \infty} \lambda_{2}(A) \geq \lim _{N \rightarrow \infty}\left|\lambda_{N}(A)\right|
$$

Now Theorem (3) is inconclusive. From $-k \leq \lambda_{N}(A) \leq 0$, we deduce that $k \leq \lambda_{N}(L) \leq 2 k$. Combining this with (11), we get the asymptotic upper bound

$$
\lim _{N \rightarrow \infty} \frac{\lambda_{2}(L)}{\lambda_{N}(L)} \leq \frac{k-2 \sqrt{k-1}}{k} .
$$

We now consider the class of Ramanujan graphs.

Definition 4 (Ramanujan Graphs): A $k$-regular graph $G=G_{N, k}$ on $N$ vertices is Ramanujan if

$$
\lambda_{G}(A) \leq 2 \sqrt{k-1} .
$$

Graphs with small $\lambda_{G}(A)$ (often called graphs with large spectral gap in the literature) are called expander graphs, and the Ramanujan graphs are one of the best explicit expanders known. Note that Theorem 3 and (10) show that, for general graphs, $\lambda_{G}(A)$ is in the limit lower bounded by $2 \sqrt{k-1}$, while for Ramanujan graphs $\lambda_{G}(A)$ is, for every finite $N$, upper bounded by $2 \sqrt{k-1}$.

From (17), it follows that, for nonbipartite Ramanujan graphs, ${ }^{3}$ the graphs of interest to us, we have the following bounds:

$$
\begin{gathered}
\lambda_{2}(A) \leq 2 \sqrt{k-1} \\
\lambda_{N}(A) \geq-2 \sqrt{k-1} .
\end{gathered}
$$

Equations (18) and (19) give, for nonbipartite Ramanujan graphs,

$$
\begin{aligned}
& \lambda_{2}(L) \geq k-2 \sqrt{k-1} \\
& \lambda_{N}(L) \leq k+2 \sqrt{k-1}
\end{aligned}
$$

and, hence, for nonbipartite Ramanujan graphs,

$$
\gamma=\frac{\lambda_{2}(L)}{\lambda_{N}(L)} \geq \frac{k-2 \sqrt{k-1}}{k+2 \sqrt{k-1}} .
$$

This is a key result. It shows that for nonbipartite Ramanujan graphs the eigenratio parameter $\gamma$ is lower bounded by (20). It will explain in what sense Ramanujan graphs are "optimal" topologies for the design problem stated in (9), as we discuss next. To show this, we compare the lower bound (20) on $\gamma$ for Ramanujan graphs with the asymptotic upper bounds (14) and (16) on $\gamma$ for generic $k$-regular graphs. We consider the two cases separately again.

1) For the family $\mathcal{G}_{1}$, the lower bound on (20) and the upper bound on (14) are the same. Since for any value of $N,(20)$ shows that $\gamma$ is above the bound, we conclude that, in the limit of large $N$, the eigenratio $\gamma$ for nonbipartite Ramanujan graphs approaches the bound from above. This contrasts with regular non-Ramanujan

${ }^{3}$ For connected nonbipartite $k$-regular graphs, the eigenvalues $\lambda_{2}(A)$, $\lambda_{N}(A)$ are nontrivial. 
graphs for which in the limit of large $N$ the eigenratio $\gamma$ stays below the bound. For the family $\mathcal{G}_{1}$ of graphs, we can then state the following optimality result.

Proposition 5: Consider the topology optimization problem for distributed inference given in (9) and with graphs restricted to the family $\mathcal{G}_{1}$. Then, as $N \rightarrow \infty$, the class of $k$-regular nonbipartite Ramanujan graphs are optimal among $\mathcal{G}_{1}$, where $k=\lfloor 2 M / N\rfloor$.

2) For the family $\mathcal{G}_{2}$, the bound (16) does not help in asserting that Ramanujan graphs have faster convergence than these generic graphs. This is because

$$
\frac{k-2 \sqrt{k-1}}{k+2 \sqrt{k-1}}<\frac{k-2 \sqrt{k-1}}{k}
$$

i.e., the lower bound (20) for Ramanujan graphs is smaller than the upper bound (16). Although for the family $\mathcal{G}_{2}$ we cannot state a result like Proposition 5, we note that the ratio of two quantities is usually much more sensitive to variations in the numerator than to variations of the denominator. Because Ramanujan graphs optimize the algebraic connectivity of the graph, i.e., $\lambda_{2}(L)$, we still expect $\gamma$ to be much larger for Ramanujan graphs than for other graphs. We show in Section IV by simulation this to be true for broad classes of graphs, including, structured graphs, small-world graphs, and Erdös-Renýi random graphs.

Regular versus nonregular graphs: Proposition 5 asserts the optimality of Ramanujan graphs for the family $\mathcal{G}_{1}$ of regular graphs. We address here the same question but contrasting regular versus nonregular graphs. We recall an inequality on the eigenratio $\gamma$ for nonregular graphs, which shows that the class of nonbipartite Ramanujan graphs are expected to perform better than many classes of nonregular graphs. Using standard spectral graph theoretic results, it can be shown that (see [15]) for any graph $G$

$$
\gamma=\frac{\lambda_{2}(L)}{\lambda_{N}(L)} \leq \frac{k_{\min }}{k_{\max }}
$$

where $k_{\min }$ and $k_{\max }$ are the minimum and maximum node degrees, respectively. Equation (21) shows that, for graphs with large heterogeneity (large spread) in degree distribution, the value of $\gamma$ is small, and, hence, such networks are not good from the point of view of distributed inference and consensus.

\section{B. Ramanujan Graphs: Explicit Algebraic Construction}

Explicit constructions of $k$-regular Ramanujan graphs (both bipartite and nonbipartite) were given independently by LubotzkyPhillips-Sarnak (LPS) [14] and Margulis [16], for the case where the degree $k$ is such that $k-1$ is a prime. The LPS construction was extended to cover the cases where $k-1$ is a prime power by Morgenstern [17]. However, recently, many probabilistic methods have been developed for constructing expander families. In particular, [18] develops a new graph product that constructs expanders of arbitrary degree and size with high probability. The nonbipartite Ramanujan graphs used in this correspondence are based on a construction given in [14], and we call them LPS-II graphs in the sequel.

LPS-II construction: The LPS-II construction gives $k$-regular nonbipartite Ramanujan graphs on $N$ vertices, where $k=p+1$ and $N=q+1$, and $p, q$ are two unequal primes congruent to $1 \bmod 4$, such that the Legendre symbol $(p / q)=1$. The LPS-II graphs are Cayley graphs over the group $P^{1}\left(F_{q}\right)=\{0,1, \ldots, q-1, \infty\}$, called the Projective line over $F_{q},{ }^{4}$ and which is basically the set of integers modulo $q$, with an additional "infinite" element inserted in it. The LPS-II graphs

\footnotetext{
${ }^{4} F_{q}$ is the field of integers modulo the prime $q$.
}

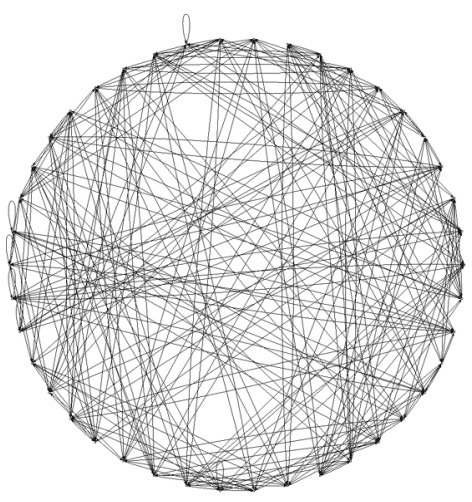

Fig. 1. LPS-II graph. Number of vertices $N=42$ and degree $k=6$.

thus obtained may have a few loops [19]; this does not pose a problem because their removal does not affect the Laplacian matrix nor its spectrum. This is because the Laplacian $L=D-A$, and a loop at vertex $n$ adds the same term to both $D_{n n}$ and $A_{n n}$, which is canceled when taking the difference. As an example of an LPS-II Ramanujan graph, we take $p=5$ and $q=41$. (It can be verified that $p, q \equiv 1 \bmod (4)$ and the Legendre symbol, $(p / q)=1$.) Thus, we have a nonbipartite Ramanujan graph, which is 6-regular and has 42 vertices. Fig. 1 shows the graph obtained.

\section{EXPERIMENTAL RESULTS}

We compare Ramanujan graphs, which are regular graphs, with regular and nonregular graphs. Define the average degree $k_{\mathrm{avg}}$ of a graph $G$ as

$$
k_{\mathrm{avg}}=\frac{2|E|}{|V|}
$$

where $|E|$ is the number of edges and $|V|$ the number of vertices in $G$. In this section, we use the symbols and terms $k$ and $k_{\text {avg }}$ interchangeably: specifically, $k$ stands for the degree in regular graphs and for the average degree in nonregular graphs. We contrast Ramanujan graphs (RG) with three classes of graphs detailed below: regular ring lattices (RRL), Watts-Strogatz small world graphs, in particular what we refer to as WS-I graphs; and Erdös-Renýi graphs. We describe briefly below the three classes of graphs used to benchmark the Ramanujan graphs.

Comparison studies: The numerical studies show the superiority of the Ramanujan graphs (RG) over the other three classes of graphs: RRL, WS-I, and ER graphs. We carry out three types of comparisons: 1) convergence speed $S_{\mathrm{c}}$ defined as $S_{\mathrm{c}}=1 / T_{\mathrm{c}}$, where the convergence time $T_{\mathrm{c}}$ is the number of iterations required for $P_{e, n}(i)$ to reach within $10 \%$ of the global probability of error $P_{e}$, averaged over all sensor nodes; 2) the $\gamma$ parameters for the RG and each of the other three classes of graphs; and 3) the algebraic connectivity $\lambda_{2}(L)$ for the RG and each of the other three classes of graphs. To simplify the comparisons, we subscript the $\gamma$ parameter by the corresponding acronym, e.g., $\gamma_{\mathrm{RG}}$ to represent the eigenratio of the Ramanujan graph. We also define the following comparison parameters

$$
\psi(\mathrm{RRL})=\frac{S_{\mathrm{c}, \mathrm{RG}}}{S_{\mathrm{c}, \mathrm{RRL}}}, \nu(\mathrm{RRL})=\frac{\gamma_{\mathrm{RG}}}{\gamma_{\mathrm{RRL}}},
$$

and

$$
\eta(\mathrm{RRL})=\frac{\lambda_{2, \mathrm{RG}}(L)}{\lambda_{2, \mathrm{RRL}}(L)}
$$

Ramanujan graphs and regular ring lattices: These are highly structured regular networks with the nodes placed on a ring, each connecting to $k / 2$ nodes to the left and $k / 2$ nodes to the right. Fig. 2 compares RG with RRL graphs. The right panel plots $\psi(\mathrm{RRL})$, the center 

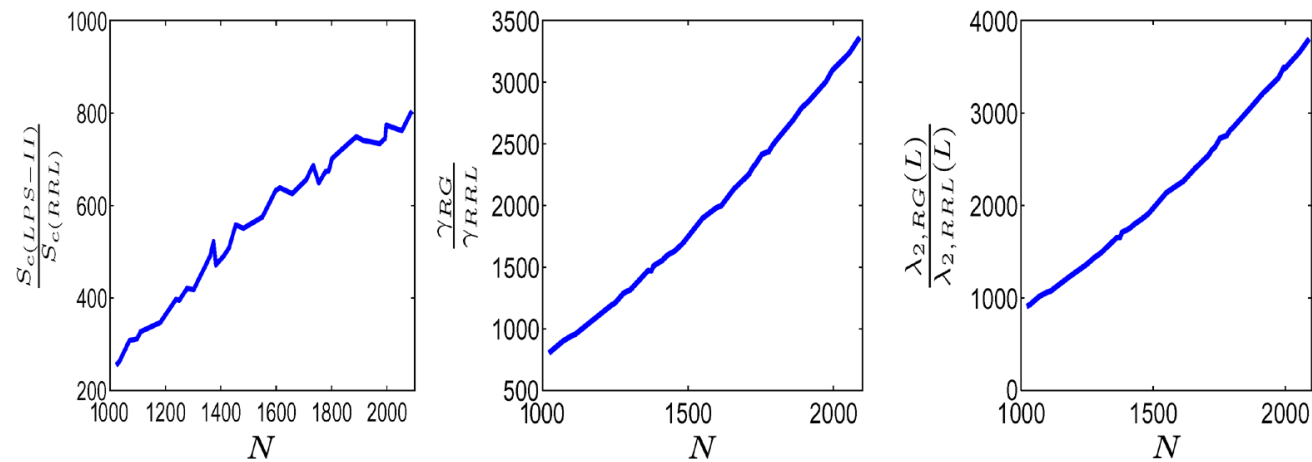

Fig. 2. Spectral properties of LPS-II and RRL graphs, $k=18$, varying $N$ : left: ratio of convergence speed $\psi(\mathrm{RRL})$; center: ratio $\nu(\mathrm{RRL})$ of $\lambda_{2}(L) / \lambda_{N}(L)$; right: ratio $\eta(\mathrm{RRL})$ of $\lambda_{2}(L)$.
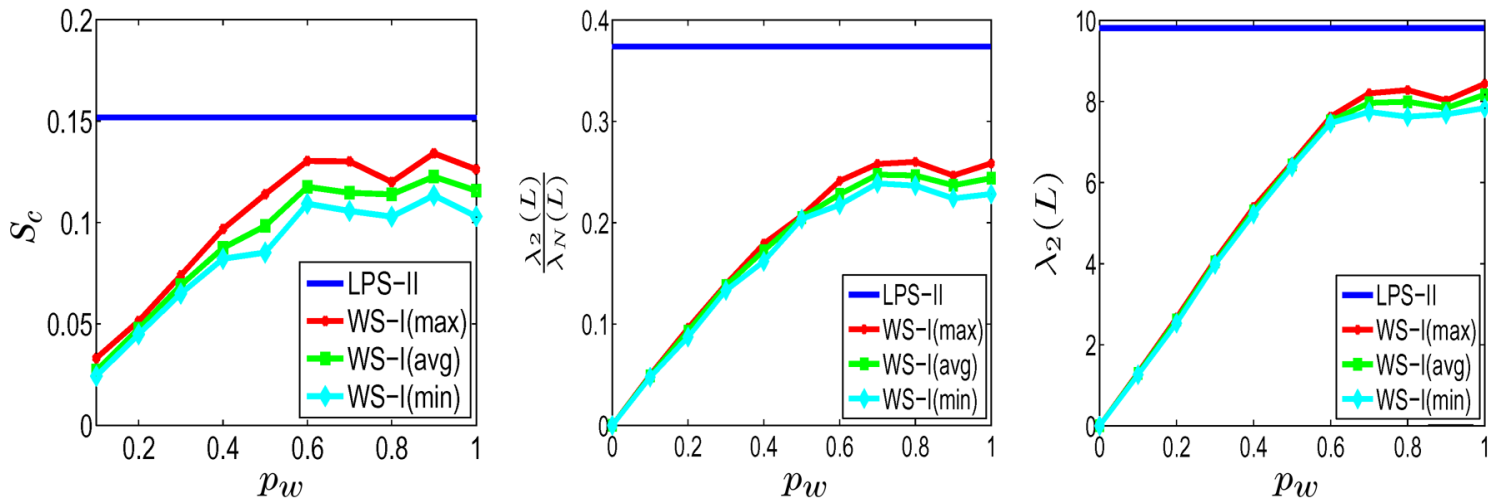

Fig. 3. Spectral properties of LPS-II and WS-I graphs, $N=6038, k=18$, varying $p_{\mathrm{w}}$ left: $S_{\mathrm{c}}$; center: eigenratio $\gamma=\lambda_{2}(L) / \lambda_{N}(L)$; right: algebraic connectivity $\lambda_{2}$.
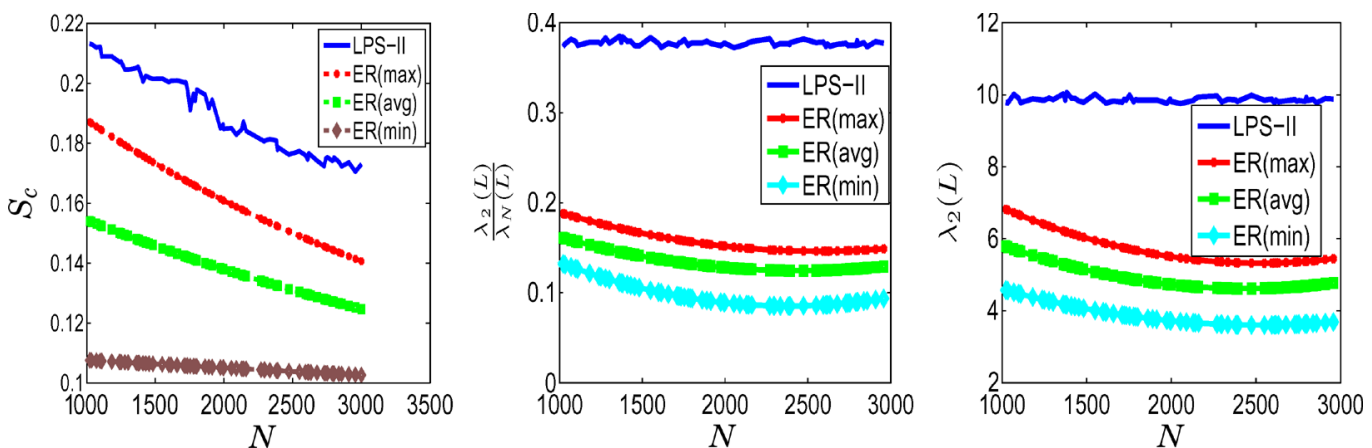

Fig. 4. Spectral properties of LPS-II and ER graphs, $k=18$, varying $N$ : left: convergence speed $S_{c}$; center: eigenratio $\gamma=\lambda_{2}(L) / \lambda_{N}(L)$; right: algebraic connectivity $\lambda_{2}$.

panel displays $\nu(\mathrm{RRL})$, and the right panel shows $\eta(\mathrm{RRL})$ when the degree $k=18$ and the number of nodes $N$ varies. The RGs converge 3 orders of magnitude faster than the RRLs, the $\gamma$ parameters can be up to 3500 times faster, and the algebraic connectivity for the RGs can be up to 4000 times larger than for the RRLs.

Ramanujan graphs and Watts-Strogatz graphs (WS-I): Small world graphs were introduced in [20]. Start with an RRL and randomly rewire all links with the same probability $p_{w}$. The resulting graph has the same number of links as the initial starting RRL seed, but may not be regular. The $p_{w}$ controls the "randomness" of the graph ( $p_{w}=0$ corresponds to the original RRL, while $p_{w}=1$ results in a random network). We call this construction the WS-I construction. In [6] and [7], we show that the WS-I graphs yield better convergence rates among the different models of small world graphs considered in that paper (WS-I, WS-II, and the Kleinberg model [21]). Hence, we restrict attention here to WS-I graphs.

The parameters are $N=6038$ and $k=18$. For WS-I, we vary $0 \leq p_{\mathrm{w}} \leq 1$. Fig. 3 shows on the left panel the convergence speed $S_{\mathrm{c}}$.
The top horizontal line is $S_{\mathrm{c}}$ for the $\mathrm{RG}$-it is flat because the graph is the same regardless of $p_{\mathrm{w}}$. The three lines below correspond to the WS-I topologies. For each value of $p_{\mathrm{w}}$, we generate $150 \mathrm{WS}-\mathrm{I}$ graphs. The top WS-I line corresponds, at each $p_{\mathrm{w}}$, to the topologies (among the 150 generated) with maximum convergence rate, the medium line to the average convergence rate (averaged over the 150 random topologies generated), and the bottom line to the topologies (among the 150 generated) with worst convergence rate. Similarly, the center and right panels on Fig. 3 compare the eigenratio parameters $\gamma$ (center panel) and the algebraic connectivity $\lambda_{2}$ (right panel). For example, the RG improves by $50 \%$ the $\gamma$ eigenratio over the best WS-I topology (in this case for $p_{\mathrm{w}}=.8$ ).

Ramanujan graphs and Erdös-Renýi graphs: These nonregular graphs randomly choose $N k / 2$ edges out of a total of $(N(N-$ 1)) $/ 2$ possible edges. Their degree distribution follows a binomial distribution, which in the limit of large $N$ approaches the Poisson law. Fig. 4 shows the results for topologies with different $N$ (horizontal axis). For each value of $N$, we generated 200 random Erdös-Renýi graphs. In the panels of both Figures, the top line illustrates the re- 


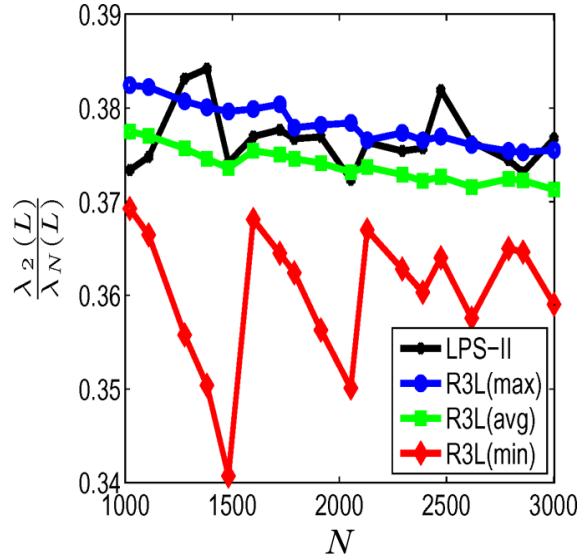

Fig. 5. LPS-II and R3L graphs, $k=18$, varying $N$ : eigenratio $\gamma=$ $\lambda_{2}(L) / \lambda_{N}(L)$.

sults for the RG, while the three lines below show the results for the Erdös-Renýi graphs-among these three, the top line is the topology with best convergence rate among the 200 ER topologies, the middle plot is the averaged convergence rate, averaged over the 200 topologies, and the bottom line corresponds to the worst topologies. Again, for example, the $\gamma$ parameter of the RG is about twice as large than the $\gamma$ parameter for the ER.

\section{RANDOM REGULAR RAMANUJAN-LIKE GRAPHS}

The RLS construction of the Ramanujan graphs restricts the values of $N$, which may limit their application in practical scenarios. We describe here briefly biased regular random graphs that can be constructed with arbitrary number of nodes $N$ and average degree, whose performance closely matches that of Ramanujan graphs. Reference [22] argues that, in general, heterogeneity in the degree distribution reduces the eigenratio $\gamma=\lambda_{2}(L) / \lambda_{N}(L)$. There exist constructions of random regular graphs, but these are difficult to implement especially for very large number of vertices, see, e.g., [23] and references therein. Our construction of random regular graphs is simple. We refer to the graphs as random regular Ramanujan-like (R3L) graphs. It starts with an arbitrary regular seed of degree $k$, e.g., an RRL with degree $k$ (see Section IV). Randomly choose (uniformly) a vertex (call it $v_{1}$.) Next, randomly choose a neighbor of $v_{1}$ (call it $v_{2}$ ), and randomly choose a vertex not adjacent to $v_{1}$ (call it $v_{3}$ ). Now choose a neighbor of $v_{3}$ (call it $v_{4}$ ). Next remove the edges between $v_{1}$ and $v_{2}$, and between $v_{3}$ and $v_{4}$. Finally, introduce edges between $v_{1}$ and $v_{3}$ and between $v_{2}$ and $v_{4}$. (Care is taken so that no conflict arises in the process of removing and forming the edges.) By construction, the graph remains $k$-regular. Repeat the steps for a sufficiently large number of times, which leads to a random regular graph with degree $k$.

Fig. 5 plots the eigenratio $\gamma=\lambda_{2}(L) / \lambda_{N}(L)$ for the RG and the R3L graphs for different values of $N$ with $k=18$. We generate $100 \mathrm{R} 3 \mathrm{~L}$ graphs for each value of $N$. The top three lines correspond to the RG, the best R3L topologies, and the average value of $\gamma$ over the $100 \mathrm{R} 3 \mathrm{~L}$ graphs. We observe that the maximum values of $\gamma=\lambda_{2}(L) / \lambda_{N}(L)$ are sometimes higher than those obtained with the LPS-II graphs. Note also that, on average, the R3L graphs are quite close to the LPS-II graphs in terms of the $\gamma=\lambda_{2}(L) / \lambda_{N}(L)$ ratio, even for large values of $N$. This confirms that the R3L graphs are a good alternative to the LPS-II graphs with the advantage that they can be generated for arbitrary number of nodes $N$ and degree $k$.

\section{CONCLUSION}

The correspondence shows that the class of nonbipartite Ramanujan graphs leads to topologies whose convergence speed in distributed in- ference and average consensus is essentially optimal for a broad class of networks. Numerical simulations verify that they outperform structured graphs, Erdös-Renýi random graphs, and small world graphs. Ramanujan graphs can be constructed only for a very restricted number of nodes. To address this limitation of LPS-II, we described a novel simple construction of random regular graphs (R3L graphs) that can have an arbitrary number of nodes $N$ and degree $k$. Simulations show that the convergence of R3L tracks very closely that of the Ramanujan LPS-II graphs. However, it should be noted that, from a network deployment point of view, the Ramanujan graphs may not be physically realizable always, for example, in a wireless communication scenario, where nodes are deployed in an ad hoc manner with nearest neighbor connectivity.

\section{REFERENCES}

[1] B. Bollobás, Modern Graph Theory. New York: Springer Verlag, 1998.

[2] R. Olfati-Saber and R. M. Murray, "Consensus problems in networks of agents with switching topology and time-delays," IEEE Trans. Autom. Control, vol. 49, no. 9, pp. 1520-1533, Sep. 2004.

[3] J. N. Tsitsiklis, "Problems in decentralized decision making and computation," Ph.D. dissertation, Massachusetts Inst. of Technology, Cambridge, MA, 1984.

[4] A. Jadbabaie, J. Lin, and A. S. Morse, "Coordination of groups of mobile autonomous agents using nearest neighbor rules," IEEE Trans. Autom. Control, vol. AC-48, no. 6, pp. 988-1001, Jun. 2003.

[5] L. Xiao and S. Boyd, "Fast linear iterations for distributed averaging," Syst. Control Lett., vol. 53, pp. 65-78, 2004.

[6] R. Olfati-Saber, "Ultrafast consensus in small-world networks," in Proc. 2005 Amer. Control Conf., Jun. 2005, vol. 4, pp. 2371-2378.

[7] S. A. Aldosari and J. M. F. Moura, "Distributed detection in sensor networks: Connectivity graph and small-world networks," in Proc. 39th Asilomar Conf. Signals, Systems, Computers, Pacific Grove, CA, Oct. 2005, pp. 230-234.

[8] M. R. Murty, "Ramanujan graphs," J. Ramanujan Math. Soc., vol. 18, no. 1, pp. 1-20, 2003.

[9] S. Kar, S. A. Aldosari, and J. M. F. Moura, "Topology for distributed inference on graphs,", Jun. 2006 [Online]. Available: http://www.arxiv. org/abs/cs/0606052, $30 \mathrm{pp}$.

[10] L. Xiao and S. Boyd, "Fast linear iteration for distributed averaging," Syst. Control Lett., vol. 53, pp. 65-78, Sep. 2004.

[11] F. R. K. Chung, Spectral Graph Theory. Providence, RI: Amer Mathematical Soc., 1997

[12] S. Kar and J. M. F. Moura, "Ramanujan topologies for decision making in sensor networks," in Proc. 44th Allerton Conf. Communication, Control, Computing, Monticello, IL, Sep. 2006.

[13] N. Alon, "Eigenvalues and expanders," Combinatorica, vol. 6, pp. $83-96,1986$

[14] A. Lubotzky, R. Phillips, and P. Sarnak, "Ramanujan graphs," Combinatorica, vol. 8, no. 3, pp. 261-277, 1988.

[15] S. Camiz and S. Stefani, Matrices and Graphs: Theory and Applications. Singapore: World Scientific, 1996.

[16] G. Margulis, "Explicit group-theoretical constructions of combinatorial schemes and their application to the design of expanders and concentrators," J. Probl. Inf. Transm., vol. 24, no. 1, pp. 39-46, 1988.

[17] M. Morgenstern, "Existence and explicit construction of $q+1$ regular Ramanujan graphs for every prime power $q$," J. Comb. Theory, ser. B, vol. 62, pp. 44-62, 1994.

[18] O. Reingold, S. P. Vadhan, and A. Wigderson, "Entropy waves, the zig-zag graph product, and new constant-degree expanders and extractors," in Proc. IEEE Symp. Foundations Computer Science, 2000, pp. $3-13$.

[19] A. Berger and J. Lafferty, "Probabilistic decoding of low-density Cayley codes," in Proc. Canadian Workshop on Information Theory, Toronto, ON, Canada, 1997.

[20] D. J. Watts and S. H. Strogatz, "Collective dynamics of small-world networks," Nature, vol. 393, pp. 440-442, 1998.

[21] J. M. Kleinberg, "Navigation in a small world," Nature, vol. 406, p. 845, Aug. 2000.

[22] A. E. Motter, C. Zhou, and J. Kurths, "Network synchronization, diffusion, and the paradox of heterogeneity," Phys. Rev. E, vol. 71, 2005.

[23] N. C. Wormald, "Models of random regular graphs," in Models of Random Regular Graphs, ser. Lecture Note Series. London, U.K.: London Mathematical Soc., 1999, vol. 267, pp. 239-298. 